\title{
On the Theory and Design of a Class of Recombination Nonuniform Filter Banks with Low-Delay FIR and IIR Filters
}

\author{
S.S.Yin, S.C.Chan and X.M.Xie \\ Department of Electrical and Electronic Engineering \\ The University of Hong Kong, Pokfulam Road, Hong Kong
}

\begin{abstract}
This paper studies the theory and design of a class of recombination nonuniform FBs (RNFB) with low-delay (LD) FIR and IIR filters. The conditions for suppressing the spurious response and achieving a good frequency characteristic for these LD FIR/IIR RNFBs are developed. The proposed LD FIR RNFBs have a lower system delay than their linear-phase counterparts, at the expense of slight increase in phase distortion of the analysis filters and arithmetic complexity. By model reducing the LD FIR uniform FBs by the modified model reduction method, an IIR RNFB with a similar characteristic can be readily obtained. A design example is given illustrate the effectiveness of the proposed method.
\end{abstract}

\section{INTRODUCTION}

Filter banks (FBs) have important applications in speech, audio, image and array processing. The theory and design of uniform perfect reconstruction (PR) and nearly perfect reconstruction (NPR) FBs have been widely studied [1]. In applications such as signal analysis and coding, FBs with nonuniform frequency spacing have the potential to offer more flexibility in time-frequency partitioning and better performance. This has attracted considerable interests in designing nonuniform FBs [2-7]. One useful approach is the indirect method proposed in [7], where certain channels of a uniform $\mathrm{FB}$ are merged using the synthesis $\mathrm{FB}$ of a recombination FB or a transmultiplexer (TMUX). The resulting FBs are called recombination nonuniform FBs (RNFBs). One advantage of the $\mathrm{RNFB}$ is that the PR property is structurally imposed as long as the original uniform and recombination filter banks are PR. Moreover, if the number of channels of the uniform and recombination TMUX are coprime, the analysis and synthesis filters of the resulting RNFB admit an equivalent linear time invariant (LTI) representation. In other words, the frequency responses of the analysis (synthesis) filters can be optimized directly, which considerably simplifies the design procedure. When the number of channels of the uniform and recombination TMUX are noncoprime, the analysis and synthesis filters are linear periodic time varying (LPTV). It was shown that Line Phase (LP) RNFB with good frequency characteristics can still be obtained even in the non-coprime case by imposing relatively simple conditions on the FBs. A class of RNFB based on the cosine modulation filter banks (CMFB) was proposed in [2]. Recently, the theory and design of RNFBs with LP filters were studied in order to achieve RNFBs with good frequency characteristic [3]. The form of the analysis LP filters is also analyzed in [3].

In this paper, the design of a class of RNFB with IIR filters is studied. Compared to FIR filters, IIR filters have the potential to offer lower system delay, sharper cutoff and higher stopband attenuation than their FIR counterparts. In [3], a class of LP RNFBs with cosine-rolloff transition band was designed using the REMEZ algorithm. It has been known that FBs designed with cosine-rolloff transition band have reasonably good reconstruction errors and the approximate power complementary $[3,8]$. In this paper, the approach in [3] is first extended to RNFBs with FIR filters having lower system delay than their LP RNFB counterparts. They are then model reduced to an IIR RNFB by using modified model reduction technique proposed in [9]. The proposed LD FIR and IIR filters have a similar frequency characteristic as the original LP analysis filters in the passband and the transition band with the group delays in these regions are slightly relaxed. As long as the stopband attenuation of the LD and LP analysis filters are comparable, reasonably good reconstruction error will be achieved. One advantage of the proposed method using the modified model reduction technique is that the stability of the model-reduced filters is guaranteed and the IIR filters obtained closely approximate the properties of the LD FIR filters. Another advantage of the proposed LD and IIR RNFBs is that they have approximately passband LP analysis filters, and hence a lower phase distortion than their IIR nonuniform CMFBs counterparts [9].

The paper is organized as follows: The theory of RNFBs is recalled in Section I. Section II is devoted to the matching condition and the design of the low delay FIR and IIR RNFBs. A design example will be given in Section III to show the efficiency of the method. Finally conclusions are drawn in Section IV.

\section{RECOMBINATION NONUNIFORM FILTER BANKS}

Figure 1 shows the structure of the RNFB considered in this paper. Consecutive channels of a uniform $M$-channel analysis FB are combined using the synthesis filters of another FB having a smaller number of channels, say $m_{k}$ as shown in the figure. The sampling rate after recombination is reduced by a factor of $\frac{m_{k}}{M}$ For simplicity, only the merging of the $m_{k}$ channels of an $M$ channel analysis bank are shown. Further merging of consecutive channels can be performed. Let the merged outputs be indexed by an integer $k, k=0, \ldots, K-1$ and $m_{k}$ the number of channels merged at the $k$-th output. For critical sampling, we have $\sum_{k=0}^{K-1}\left(m_{k} / M\right)=1$. In the synthesis banks, each merged output will pass through the analysis filters of the recombination FBs and they will be fed to the synthesis bank of the uniform FB for reconstruction. Each synthesis-analysis structure, involving an $m_{k}$ channel $\mathrm{FB}$, is called a TMUX. It was observed in [2] that if the TMUXs are PR, then they only introduce a signal delay in the subband signal of the uniform FB. If these delays are properly 
compensated in other subbands, then the system is PR if the original uniform FB is PR. Further, if $m_{k}$ and $M$ are coprime to each, then the equivalent analysis filter of the $k$-th output is LTI. In the more general case, the analysis filters are LPTV as $m_{k}$ and $M$ are non-coprime. The discrete-time Fourier transform (DTFT) of the $k$-th merged output can be written as:

$$
\begin{aligned}
Y_{k}\left(e^{j \omega}\right)= & \frac{1}{M} \sum_{i=0}^{m_{k}-1} G_{k, i}\left(e^{j \omega}\right) \\
& \times \sum_{\tau=0}^{M-1} H_{l_{k}+i}\left(e^{j \frac{m_{k}(\omega-2 \pi \tau}{M}}\right) X\left(e^{j \frac{m_{k}(\omega-2 \pi \tau}{M}}\right),
\end{aligned}
$$

where $X\left(e^{j \omega}\right)$ and $Y_{k}\left(e^{j \omega}\right)$ are the input and the $k$-th subband output of the RNFB. To save notation, we shall write $X(\boldsymbol{a})$ as $X\left(e^{j \omega}\right), Y_{k}(a)$ as $Y_{k}\left(e^{j \omega}\right)$ for the input, output and other related quantities. Here we assume that the merging of the $m_{k}$ - channel TMUX starts at the $l_{k}$-th channel of the $M$-channel uniform FB. (1) can be written as:

$$
Y_{k}(\omega)=\frac{1}{M} \sum_{\tau=0}^{m_{k}-1} A_{\tau}(\omega) X\left(\left(m_{k} \omega-2 \pi \tau\right) / M\right)
$$

where

$$
A_{\tau}(\omega)=\sum_{i=0}^{m_{k}-1} G_{k, i}(\omega) H_{l_{k}+i}\left(\left(m_{k} \omega-2 \pi \tau\right) / M\right),
$$

$\tau=0, \ldots, M-1 . A_{\tau}(a)$ should be designed to extract the frequency components of $X(a)$ exactly. It is known that the TMUX can be derived from an 1-skewed FB [10]. Let $\left\{G_{i}^{\prime}(z), G_{i}(z)\right\}$, $i=0, \ldots, m_{k}$, be the analysis filters and the synthesis filters of a uniform FB. The 1-skewed FB with the analysis filters and synthesis filters $\left\{G_{k, i}^{\prime}(z), G_{k, i}(z)\right\}$ satisfy $G_{k, i}^{\prime}(z)=G_{i}^{\prime}(z)$ and $G_{k, i}(z)=z^{-1} G_{i}(z)$. For simplicity, we define $\bar{H}_{l_{k}+i}(\omega)=H_{l_{k}+i}\left(m_{k} \omega / M\right)$. So (3) can be written as:

$$
A_{\tau}(\omega)=e^{-j \omega} \sum_{i=0}^{m_{k}-1} G_{i}(\omega) \bar{H}_{i_{k}+i}\left(\omega-2 \pi \tau / m_{k}\right),
$$

$\tau=0, \ldots, M-1$. Note that $\bar{H}_{l_{k}+i}(\omega)$ and $G_{i}(\omega)$ can be written as:

$$
\begin{aligned}
& \bar{H}_{l_{k}+i}(\omega)=\bar{H}_{l_{k}+i}^{+}(\omega)+\bar{H}_{l_{k}+i}^{-}(\omega),-\pi M / m_{k} \leq a \leq \pi M / m_{k} \\
& G_{i}(\omega)=G_{i}^{+}(\omega)+G_{i}^{-}(\omega),-\pi / M \leq a \leq \pi / M .
\end{aligned}
$$

Assume that the stopband edges of $\bar{H}_{l_{k}+i}(\omega)$ and $G_{i}(\omega)$ lie within $\left[\pi\left(l_{k}+i\right) / M-\pi / 2 M, \pi\left(l_{k}+i \dashv 1\right) / M+\pi / 2 M\right] \quad$ and $\left[\pi i / m_{k}-\pi / 2 m_{k}, \pi(i+1) / m_{k}+\pi / 2 m_{k}\right]$, respectively, and the stopband attenuation is sufficiently high that it can be treated as zero outside the region. Let's consider the right hand side of (4) without the trivial delay:

$$
\begin{aligned}
D(\omega) & =\sum_{i=0}^{m_{k}-1} G_{i}(\omega) \bar{H}_{l_{k}+i}\left(\omega-2 \pi \tau / m_{k}\right) \\
& =D_{1}(a) \dashv D_{2}(a) \dashv D_{3}(a)+D_{4}(a),
\end{aligned}
$$

where

$$
\begin{aligned}
& D_{1}(\omega)=\sum_{i=0}^{m_{k}-1} G_{i}^{+}(\omega) \bar{H}_{l_{k}+i}^{+}\left(\omega-2 \pi \tau / m_{k}\right), \\
& D_{2}(\omega)=\sum_{i=0}^{m_{k}-1} G_{i}^{-}(\omega) \bar{H}_{l_{k}+i}^{+}\left(\omega-2 \pi \tau / m_{k}\right)
\end{aligned}
$$

$D_{3}(a)$ and $D_{4}(a)$ conjugate of $D_{1}(a)$ and $D_{2}(a)$. Define $|q| \leqslant\left\lfloor M / m_{k}\right\rfloor$ and $|\gamma|=\left\lfloor l_{k} / 2\right\rfloor$, where $\lfloor x\rfloor$ denotes the nearest integer less than or equal to $x$. In order to determine the spurious response, passband and stopband of the various components, let's examine the difference between the starting locations of the passband edges of the supports of $\bar{H}_{l_{k}+i}(\omega)$ and $G_{i}(\omega)$. It can be seen that all components in $D_{1}(a)$ constitute the passband of the LPTV filter $A_{\tau}(a)$ if $\tau=q m_{k}-\gamma$. However, $D_{2}(a)$ will probably give rise to undesirable spurious response from the overlapping of the transition bands of $\bar{H}_{l_{k}+i}(\omega)$ and $G_{i}(\omega)$. The source of the spurious response, which is the cross-term in $D_{2}(a)$, causes bumping in the stopband of $A_{\tau}(a)$. Fortunately, it will be shown below, similar to the result in [3] for the RNFBs with LP filters, that the spurious response can be suppressed if the appropriate conditions on the constant $c_{i}$ and a matching condition on the LD FIR or IIR analysis/synthesis filters are imposed.

\section{RECombination NonUNiform Filter Banks WITH LD-FIR AND IIR FILTERS}

In the proposed $\mathrm{FB}$, the analysis filters are either LD FIR or IIR filters. For the case of LD FIR filters with filter order $N$, its frequency response can be written as $H\left(e^{j \omega}\right)=e^{-j \omega D / 2} H_{R}(\omega)$, where $H_{R}(\omega)$ is a complex function. If $H_{R}(\omega)$ approximates a real function, then it represents approximately the magnitude response and the passband group delay is approximately constant and is equal to $(D / 2)$, which can be made smaller than $(N / 2)$ for its LP counterpart. In the latter case, $H_{R}(\omega)$ is real-valued. Further, an LD FIR filter can be made approximately symmetry/antisymmetry if the first $(D+1)$ samples of its impulse response are approximately symmetry/anti-symmetry. If filters $\bar{H}_{l_{k}+i}(\omega)$ and $G_{i}(\omega)$ in the LD RNFB are approximately symmetric, then their frequency response can be written as:

$$
\bar{H}_{l_{k}+i}(\omega) \approx e^{-j \alpha \omega} \bar{H}_{l_{k}+i}^{R}(\omega), G_{i}(\omega) \approx e^{-j \beta \omega} G_{i}^{R}(\omega) .
$$

Similar, if $\bar{H}_{l_{k}+i}(\omega)$ and $G_{i}(\omega)$ are approximately anti-symmetric:

$$
\begin{aligned}
& \bar{H}_{l_{k}+i}^{+}(\omega) \approx j e^{-j \alpha \omega} \bar{H}_{l_{k}+i}^{R}(\omega), \bar{H}_{l_{k}+i}^{-}(\omega) \approx-j e^{-j \alpha \omega} \bar{H}_{l_{k}+i}^{R}(\omega), \\
& G_{i}^{+}(\omega) \approx j e^{-j \beta \omega} G_{i}^{R}(\omega), G_{i}^{-}(\omega) \approx-j e^{-j \beta \omega} G_{i}^{R}(\omega),
\end{aligned}
$$

where, $\bar{H}_{l_{k}+i}^{R}(\omega)$ and $G_{i}^{R}(\omega)$ are the corresponding amplitude responses, $a$ and $\beta$ are the passband group delay. Due to space limitation, we only consider LD FBs with filters that are symmetric when $i$ is even and anti-symmetry when $i$ is odd. The cross-term in $D_{2}(a)$, which is the source of the spurious response and causes the bumps in the stopband of $A_{\tau}(a)$, becomes:

$$
\begin{aligned}
& e^{-j(\alpha+\beta) \omega}\left(c_{l_{k}+i} \bar{H}_{l_{k+i}}^{R}\left(\omega-2 \pi \tau / m_{k}\right) G_{i}^{R}(\omega)\right. \\
& \left.\quad+c_{l_{k}+i+1} \bar{H}_{l_{k}+i+1}^{R}\left(\omega-2 \pi \tau / m_{k}\right) G_{i+1}^{R}(\omega)\right)
\end{aligned}
$$

The cross-term can be cancelled if we impose the condition on the constants $c_{i}: c_{l_{k}+i}=-c_{l_{k}+i+1}$, when the uniform LD FB and the recombination LD TMUX are designed to satisfy (the matching condition):

$$
\bar{H}_{l_{k}+i}(\omega)=G_{i}(\omega)
$$

which also can be written as: 


$$
H_{l_{k}+i}\left(m_{k} \omega / M\right)=G_{i}(\omega),
$$

for $a \in\left[-\left(\pi / m_{k}\right),\left(\pi / m_{k}\right)\right]$. On the other hand:

$$
\begin{aligned}
D_{1}(\omega)= & \sum_{i=0}^{m_{k}-1} c_{l_{k}+i} G_{i}^{+}(\omega) \bar{H}_{l_{k}+i}^{+}\left(\omega-2 \pi \tau / m_{k}\right) \\
\approx & e^{-j(\alpha+\beta) \omega}\left(c_{l_{k}} \bar{H}_{l_{k}}^{R}\left(\omega-2 \pi \tau / m_{k}\right) G_{0}^{R}(\omega)\right. \\
& \left.\quad-c_{l_{k}+1} \bar{H}_{l_{k}+1}^{R}\left(\omega-2 \pi \tau / m_{k}\right) G_{1}^{R}(\omega)+\cdots\right) .
\end{aligned}
$$

Therefore, the constants $c_{i}$ need to satisfy: $c_{l_{k}+i}=-c_{l_{k}+i+1}$ in order to achieve a flat passband.

In the design of the uniform LD FB, we use remez algorithm in Matlab. The ideal magnitude responses of all analysis filters in original FB and TMUXs have the form of a cosine function:

$$
\begin{aligned}
& H_{0}(\omega)=\left\{\begin{array}{cc}
1 & 0 \leq \omega \leq \omega_{1_{-} p 2} \\
\cos \left(\frac{\pi}{2 \Delta \omega}\left(\omega-\omega_{1_{-} p 2}\right)\right) & \omega_{1_{-} p 2} \leq \omega \leq \omega_{1_{s} s} \\
0 & \omega_{1_{-} s 2} \leq \omega \leq \pi
\end{array}\right. \\
& H_{k}(\omega)=\left\{\begin{array}{cl}
0 & \leq \leq \leq \omega_{k_{-} s 1} \\
\cos \left(\frac{\pi}{2 \Delta \omega}\left(\omega_{k_{-} p 1}-\omega\right)\right) & \omega_{k_{-} s 1} \leq \omega \leq \omega_{k_{-} p 1} \\
1 & \omega_{k_{-} p 1} \leq \omega \leq \omega_{k_{-} p 2}, k=1, \ldots, M-2 \\
\cos \left(\frac{\pi}{2 \Delta \omega}\left(\omega-\omega_{k_{-} p 2}\right)\right) & \omega_{k_{-} p 2} \leq \omega \leq \omega_{k_{-} s 2} \\
0 & \omega_{k_{-} s 2} \leq \omega \leq \pi
\end{array}\right. \\
& H_{M-1}(\omega)=\left\{\begin{array}{cc}
0 & 0 \leq \omega \leq \omega_{M-1 \_s 1} \\
\cos \left(\frac{\pi}{2 \Delta \omega}\left(\omega_{M-1 \_p 1}-\omega\right)\right) & \omega_{M-1 \_s 1} \leq \omega \leq \omega_{M-1 \_p 1} \\
1 & \omega_{M-1 \_p 1} \leq \omega \leq \pi
\end{array},\right.
\end{aligned}
$$

where, for $i=1, \ldots, M-1, a_{i_{-} p 1}=(i \pi / M)+(\Delta a / 2)=a_{i-1 \_s 2}$ and $a_{i_{-} s 1}=(i \pi / M)-(\Delta a / 2)=a_{i-1 \_p 2}$ are the cutoff frequencies; and for $k=1, \ldots, M-2$, the transition bandwidth is: $\Delta a=a_{k_{-} s 2}-a_{k_{-} p 2}=a_{k_{-} p 1}-a_{k_{-} s 1}$. The transition bandwidth also includes $\Delta a=a_{0_{-} s 2}-a_{0_{-} p 2}=a_{M-11_{-} p 1}-a_{M-1 \_s 1}$. The FB so obtained is approximately power complementary and NPR.

By applying the modified model reduction approach proposed in [9] to the FIR RNFB, a causal and stable IIR RNFB with a similar frequency characteristic will be obtained. The polyphase components of the IIR filter so obtained have identical denominator [9] in the form of:

$$
P_{k}(z)=\frac{N_{k}(z)}{D(z)}, k=0, \ldots, M-1 \text {. }
$$

This simplifies the realization of the IIR filters in the FB because the polyphase components have identical denominator. The optimal denominator $D(z)$ can be determined using the following iterative procedure (interested reader can refer [9] for more detail):

$$
D^{(k)}(z)=1+\sum_{n=1}^{L_{q}-1} q^{(k)}(n) z^{-n}, D^{(0)}(z)=1 .
$$

Another advantage of the modified model reduction is that the denominator and the numerator can be determined separately. In addition, the model reduced IIR filters are always stable and their frequency responses closely resemble the original FIR filters.

\section{EXAMPLE}

A design example is given to illustrate the efficiency of the proposed method in designing LD FIR and IIR RNFBs. An LD FIR RNFB is designed with the following parameters: $M=6$, $m_{0}=3, m_{1}=2$. Hence the decimation factors are $\{3 / 6,1 / 6,2 / 6\}$.
Note that since the rate- $1 / 2$ and the rate-1/3 do not satisfy the coprime condition, the analysis filters are LPTV. The original LD FB and recombination LD TMUXs are designed separately as according to the existence condition on $c_{i}$ and the matching condition. We choose $c_{l_{k}+i}=1$ for even $i$ and $c_{l_{k}+i}=-1$ for odd $i$. The filters were designed to approximate the cosine-rolloff property described in (12). As an illustration, the 6-, 3- and 2channel uniform LD FIR FBs with respective system delays $D$ 's of 109,50 and 33 samples satisfying the matching conditions in (9) are designed and then model reduced to obtain an IIR RNFB. Here, the length $L$ of the analysis filters of the 6-, 3- and 2-channel uniform LD FIR FBs are respectively chosen as $(126,63,42)$. Whereas the parameters (length of numerator, length of denominator, number of non-zero coefficients of the denominator, excluding the first coefficient, $d$ ) of the model-reduced prototype filters of the 6-, 3- and 2-channel uniform IIR FBs are respectively chosen as $(114,31,5),(57,7,2)$ and $(38,3,1)$. Table 1 summarizes the above design parameters.

Table 1. filter lengths and group delays in Figures 2(a)-(d)

\begin{tabular}{|c|c|c|c|}
\hline & $\begin{array}{c}6 \text {-channel } \\
\text { FB }\end{array}$ & $\begin{array}{c}\text { 3-channel } \\
\text { TMUX }\end{array}$ & $\begin{array}{c}\text { 2-channel } \\
\text { TMUX }\end{array}$ \\
\hline Lengths of LD FIR filters: & 126 & 63 & 42 \\
\hline $\begin{array}{c}\text { Length of numerator of } \\
\text { IIR filters }\end{array}$ & 114 & 57 & 38 \\
\hline $\begin{array}{c}\text { Length of denominator of } \\
\text { IIR filters }\end{array}$ & 31 & 7 & 33 \\
\hline System delays & 109 & 50 & 3 \\
\hline
\end{tabular}

The proposed LD FIR and IIR filters have a similar frequency characteristic as the LP analysis filters (in [3]) in the passband and the transition band, except that the group delays in these regions are slightly relaxed in exchange for a lower system delay and slightly lower filter order. It can be seen that as long as the stopband attenuation of the LD and LP analysis filters are comparable, reasonably good reconstruction error will be achieved by means of the existence and matching conditions. Since the number of channels of the FB and the TMUXs are not coprime, the analysis filters of the above FB are LPTV. Figures 2(a)-( $f$ ) show the frequency responses of the LPTV analysis filters so obtained. The amplitudes and phases of all frequency components are generated by imputing a sinusoidal signal at certain frequency with unit amplitude and zero phase.

\section{Conclusions}

The theory and design of a class of RNFBs with LD FIR and IIR filters are presented. The conditions for suppressing the spurious response and achieving a good frequency characteristic are developed. The proposed LD FIR RNFBs have a lower system delay than their LP counterparts. By model reducing the LD FIR analysis/synthesis filters by the modified model reduction method, an IIR RNFB with a similar characteristic can be readily obtained. A design example is given illustrate the effectiveness of the proposed method.

\section{REFERENCES}

[1] P. P. Vaidynathan, Multirate systems and filter banks, Englewood cliffs, NJ: Prentice Hall, 1992.

[2] X. M. Xie, S. C. Chan and T. I. Yuk, "Design of perfectreconstruction nonuniform recombination filter-banks with flexible rational sampling factors," IEEE Trans. on CAS-I, vol.52, no. 9, pp. 1965-1981, Sept 2005. 
[3] X. M. Xie and S. C. Chan, "The theory and design of a class of recombination nonuniform filter banks with linear phase filters," IEICE Trans.INF.\&SYST., vol. E88-D, no. 7, pp. 1445-1451, July. 2005.

[4] F. Argenti and E.Del Re, "Rational sampling filter bansks based on IIR filters," IEEE Trans. on SP., vol. 45, no. 12, pp. 3403-3408, Dec. 1998.

[5] S. Akkarakaran and P. P. Vaidyanathan, "New results and open problems on nonuniform filter-banks," in Proc. IEEE ICASSP'99, vol. 3, pp. 1501-1504, March 1999.

[6] J. Kovacevic and M. Vetterli, "Perfect reconstruction filter banks with rational sampling factors," IEEE Trans. on SP., vol. 41, pp.20472066, Jun. 1993
[7] R. V. Cox, "The design of uniformly and nonuniformly spaced pseudoquadrature mirror filters," IEEE Trans. on ASSP., vol. ASSP24, pp. 1090-1096, Oct. 1986.

[8] S. C. Chan and S. S. Yin, "On the design of low delay nearly-PR and PR FIR cosine modulated filter banks having approximate cosine rolloff transition band," in Proc. EUSIPCO 2004., pp. 1253-1256, Sept. 2004

[9] S. C. Chan and S. S. Yin, "On the theory and design of a class of PR causal-stable IIR Non-uniform Recombination Cosine Modulated Filter Banks," in Proc. IEEE ISCAS 2005., pp. 1094-1097, May 2005.

[10] R. D. Koilpillai, T. Q. Nguyen and P. P. Vaidynathan, "Some results in the theory of crosstalk-free transmultiplexers," IEEE Trans on SP, vol. 39 , no. 10 , pp. 2174-2183, Oct. 1991.

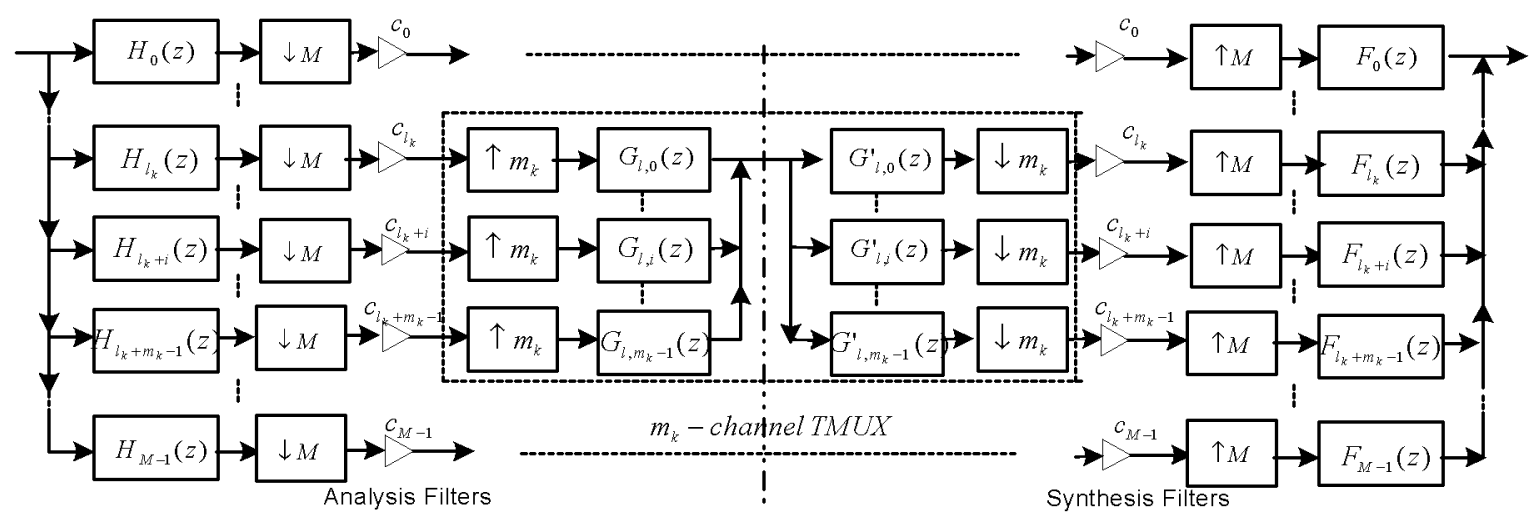

Figure 1 Structure of recombination nonuniform filter bank

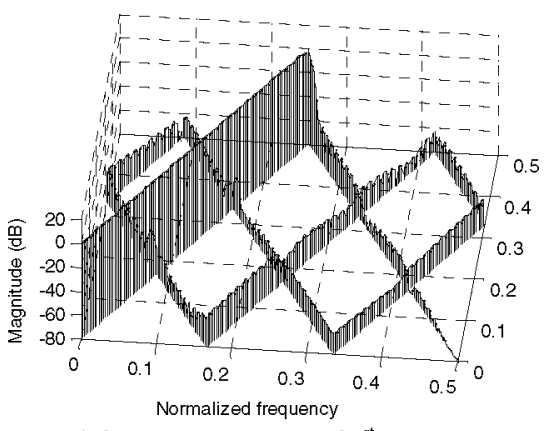

(a) Bi-frequency response of $1^{\text {st }}$ LD FIR LPTV analysis filter

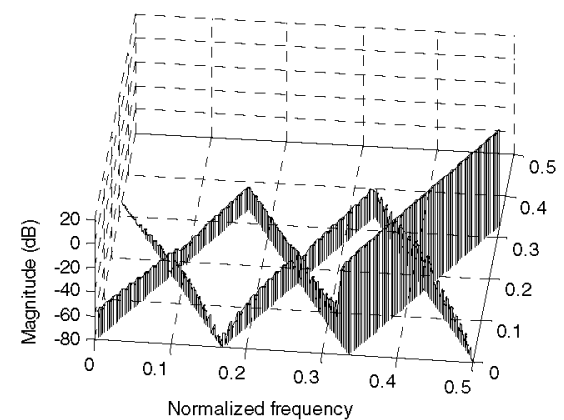

(b) Bi-frequency response of $3^{\text {rd }}$ LD FIR LPTV analysis filter

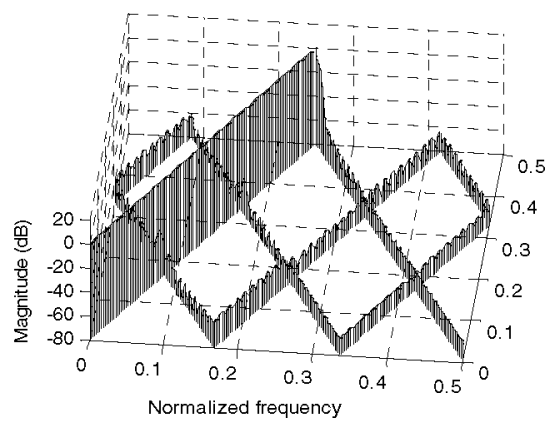

(c) Bi-freuency response of $1^{\text {st }}$ IIR LPTV analysis filter

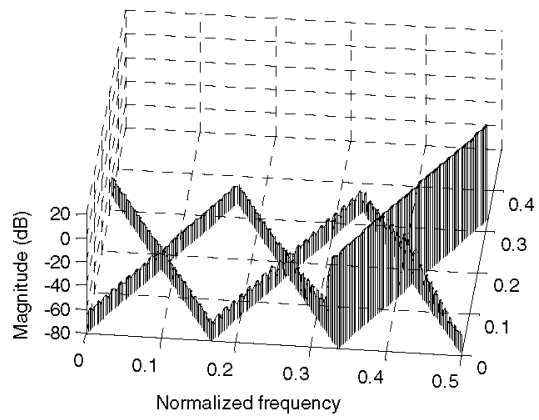

(d) Bi-frequency response of $3^{\text {rd }}$ IIR LPTV analysis filter

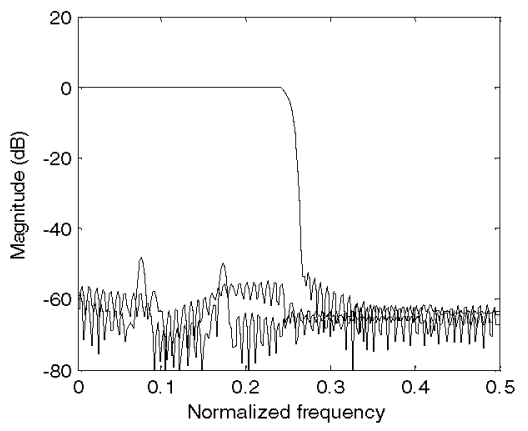

(e) the projection of (c) on the digital frequency axis

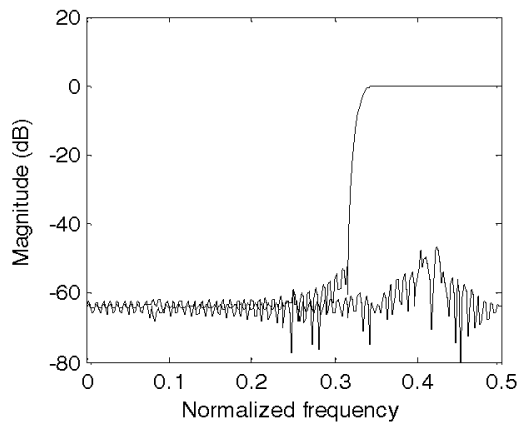

(f) the projection of (d) on the digital frequency axis

Figure 2. Responses of $1^{\text {st }}$ and $3^{\text {rd }}$ analysis filters in LD FIR and IIR RNFB with sampling rate $\{3 / 6,1 / 6,2 / 6\}$. 